\title{
Secondary structure prediction of beta-hairpin peptide tryptophan zipper-I
}

\author{
Gökhan Gökoğlu ${ }^{\mathrm{a}, \mathrm{b}, *}$, Tarık Çelik $^{\mathrm{c}}$ \\ ${ }^{a}$ Karabük University, Department of Physics, 78100, Karabük, Turkey \\ ${ }^{\mathrm{b}}$ Bilkent University, Department of Physics, 06800, Bilkent, Ankara, Turkey \\ ${ }^{\mathrm{c}}$ Turkish Academy of Sciences, Piyade Sokak No: 27, 06550, Çankaya, Ankara, Turkey \\ Received 3 May 2007 \\ Available online 4 March 2008
}

\begin{abstract}
We have investigated the folding properties of tryptophan zipper-I molecule which folds into a stable $\beta$-hairpin motif in aqueous solution as suggested by nuclear magnetic resonance (NMR) experiments. An all-atom presentation, including hydrogen, was used with an implicit solvent. As a simulation technique, simulated tempering algorithm was used to obtain equilibrium conformations of the molecule at ten distinct temperatures. Our minimum energy configuration obtained from simulated tempering algorithm is a $\beta$-hairpin motif with $1.30 \AA ̊$ backbone root-mean-square deviation from the reference PDB structure (1le0.pdb). Several quantities and exhaustive folding free energy landscapes were determined and discussed in order to clarify the folding behavior.
\end{abstract}

(C) 2008 Elsevier B.V. All rights reserved.

PACS: 02.70.Uu; 87.15.Cc; 82.20.Wt

Keywords: Tryptophan zipper; $\beta$-hairpin; Generalized ensembles; Simulated tempering

\section{Introduction}

The major goal of protein folding studies is to determine the stable structure and to investigate the conformational properties of the system. The determination of the 3D structure of biological macromolecules has attracted great interest for the last two decades among the general aspects of the protein folding problem. Numerous simulations of the short peptides have been carried out to find answers to the central issues of the folding behavior of proteins [1-3]. The studies of small proteins with well-defined tertiary structures improve our understanding of the folding principles of larger proteins. Peptide chains which fold into definite motifs like $\alpha$-helices or $\beta$-hairpins are specially preferred for theoretical investigations in order to see whether the suggested model or computational technique works well due to verification necessities with experimental results.

The problem of protein folding entails the study of a non-trivial dynamics along pathways embedded in a rugged energy landscape. The topology of the landscape characterizes the folding kinetics through the existence of a folding

\footnotetext{
* Corresponding author at: Karabük University, Department of Physics, 78100, Karabük, Turkey. Tel.: +90 3122902151; fax: +90 3122664579.

E-mail addresses: ggokoglu@fen.bilkent.edu.tr (G. Gökoğlu), tcelik@ tuba.gov.tr (T. Çelik).
} 
pathway. There are several force fields and simulation algorithms that can be used in the prediction of secondary and tertiary structures of peptides and proteins, in view of various models [4-6]. Realistic physical models based on an all-atom description of polypeptides give very detailed information about folding dynamics but computationally it is extremely demanding $[7,8]$. This is one of the main reasons that small polypeptide chains were usually preferred in all-atom simulations. Coarse grained lattice and off-lattice models allow for systematic thermodynamic studies and partly sequence analysis of polymers up to 100 monomers $[9,10]$. However, introducing the more refined potential functions gives a chance to study the larger systems without ignoring the important physical interactions.

Trpzip-I molecule, a 12-residue small peptide, forms a stable $\beta$-hairpin structure in aqueous solution [11]. Moreover, NMR data of that molecule is unusually of high quality for a short peptide indicating a highly ordered structure. The main goal of this study is to determine the stable equilibrium conformations and to construct the free energy landscape of this molecule by using a generalized ensemble Monte Carlo algorithm, simulated tempering, in order to discuss the folding behavior.

The application of the simulated tempering algorithm to biological systems is relatively new [12]. Other generalized ensemble techniques have been applied to polypeptide systems with diverse folds. The numerical performance of simulated tempering algorithm was proved to be equivalent to that of the sophisticated generalized ensemble technique multicanonical Monte Carlo using a five-residue small peptide Met-enkephalin [13]. The reason we utilize the simulated tempering method in sampling of the conformational space is to figure out the efficiency of this technique in the folding of a beta-hairpin peptide with rather complex free energy landscape.

\section{Method}

In this study, we have used an all-atom presentation based on local, excluded volume, hydrogen bond and hydrophobic interactions with an implicit solvent potential energy implemented into a newly introduced program package PROFASI (PROtein Folding and Aggregation SImulator) [14]. Peptides with 20 amino acids have been successfully studied with this potential energy function and program package $[15,16]$. There are also other folding and optimization packages based on Monte Carlo techniques with open codes like SMMP [17], GROMACS [18], and FANTOM [19]. SMMP code contains various generalized ensemble algorithms and performs calculations on the basis of ECEPP force field with different atomic solvation parameter sets for implicit solvent approximation. But this force field fails to fold peptides containing large amount of $\beta$-structures in native folds. This is mainly due to the optimization of the empirical force field parameters from crystal structures. Contrary, its success is remarkable in predicting the helical structures. PROFASI uses a simplified energy function which was calibrated by considering a set of well-characterized peptides. Consequently, it provides better handling of peptides folding into different secondary structures.

In the studies of complex systems, conventional simulation methods are not effective because the system often becomes trapped for long simulation times in a potential well. Consequently, the equilibrium configurations of the system cannot be constructed accurately through a Markov chain. The trapping problem of Metropolis Monte Carlo and Molecular Dynamics methods can be alleviated to a large extent by generalized ensemble algorithms.

There are several generalized ensemble algorithms and global optimization techniques like multicanonical algorithm, entropic sampling, simulated annealing, parallel tempering, and simulated tempering; all of these algorithms were applied to small protein and polypeptide systems to investigate the structural and thermodynamic properties in previous studies [20-22]. Mathematical similarities of multicanonical algorithm and entropic sampling were explained in Ref. [23]. Multicanonical ensemble is based on a probability function in which different energies are equally probable [24]. Multicanonical weights $\omega(E) \sim 1 / n(E)$, where $n(E)$ is the density of states, are unknown and calculated at the first stage of the simulation by an iterative procedure. The full simulation is performed with fixed weights and the resulting multicanonical sampling displays a flat histogram distribution according to energy. In simulated annealing algorithm, the sampling starts with a Metropolis simulation at high temperature and temperature is regarded as a global parameter [25]. At high temperatures, the probability to accept a conformation is relatively high due to low energy barriers. Then the simulation temperature is decreased gradually until it reaches the final temperature; the system is annealed and the atoms are enabled to relax into stable states. In parallel tempering algorithm, several Metropolis Monte Carlo simulations were performed at distinct temperatures [26]. Exchanges of conformations were periodically realized between non-interacting replicas and the system is not trapped as in Metropolis, due to global updates via replica exchanges which thermalize the system fast to give a true canonical 


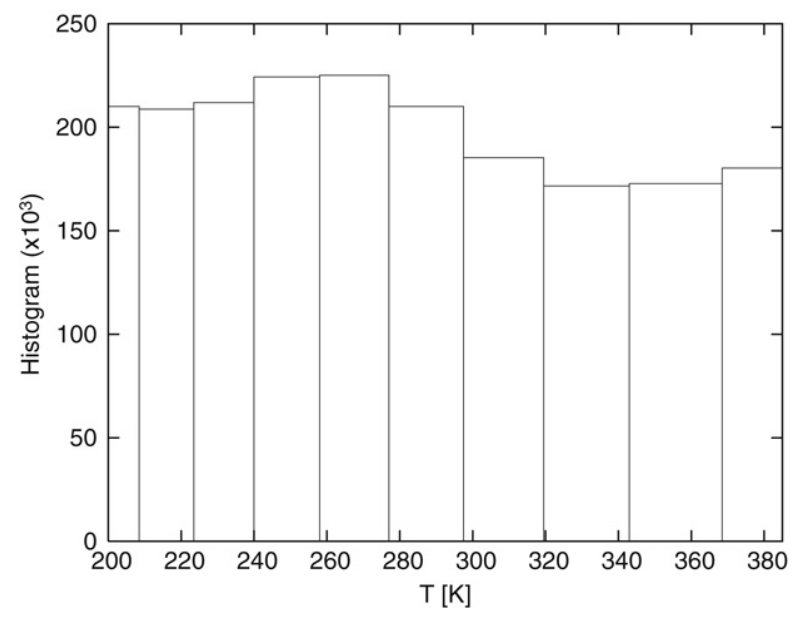

Fig. 1. Number of visits to different temperatures in simulated tempering run.

distribution at each temperature copy [27]. The efficiency of this technique is high and implementation is simple. Application of this technique to biological macromolecules was reviewed in a previous work [28].

The main idea of the simulated tempering algorithm is to change the temperature of the system while remaining at equilibrium [29]. In that sense, it differs from the simulated annealing in which the system goes away from the equilibrium state while changing the temperature. As a generalized ensemble technique, simulated tempering algorithm $[12,30]$ simulates the ensemble by partition function;

$$
Z=\sum_{i} \exp \left(-g_{i}\right) Z\left(T_{i}\right)
$$

where $g_{i}$ 's are the tunable parameters which are determined by an iterative procedure through trial runs and control the jumping to different temperatures. Configuration and temperature updates are realized according to the weight function [13];

$$
\omega(T, E)=\exp (-E / T-g(T))
$$

which leads to a constant probability distribution for temperature.

Our simulated tempering procedure is composed of 10 different temperatures between 200 and $380 \mathrm{~K}$ with $T_{i}=T_{\min }\left(T_{\max } / T_{\min }\right)^{(i-1) / 9}$. In simulated tempering algorithm, an exponential distribution of temperatures is chosen in the similar form to our expression [15,22]. Uniform distribution of temperatures does not fulfill the reliable calculation of estimators $g_{i}(T)$. This situation is directly related to the Metropolis criteria and the tunneling probability to neighboring temperatures.

In Fig. 1, we have shown the number of sampled conformations for each temperature studied in this work. The number of sampled conformations are very close for each temperature indicating that a flat histogram distribution is achieved, which has central importance for the generalized ensemble algorithms and is a major indication of the computational efficiency of this technique. Temperature is the dynamical global quantity in this technique. Jumping to different temperatures is only possible for neighboring temperatures which is realized according to improved Metropolis criteria explained in Ref. [29]. We have shown the Monte Carlo time series in Fig. 2. Time series is an important characteristic for any Monte Carlo technique and reflects the general behavior of the algorithm. Our time series shown in the figure is a typical one for simulated tempering [13]. The simulation reaches the global minimum energy valley at $\sim 246,000$ sweeps. It is obvious that, this value strongly depends on the complexity of the energy surface. The system under study, tryptophan zipper-I molecule, is composed of 220 atoms. Relatively better results can be obtained by smaller peptide chains. Our simulation contains $2 \times 10^{6}$ Monte Carlo cycles in total. This reflects high statistics for effective search of conformational space. 


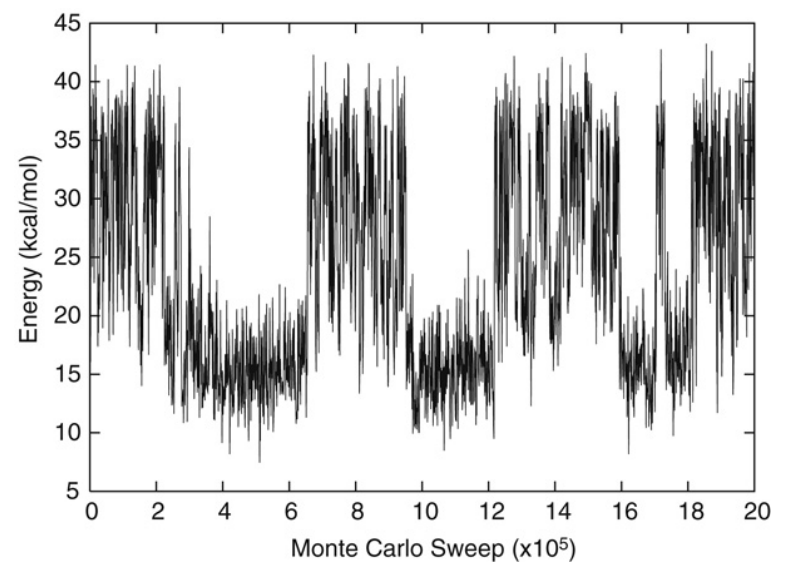

Fig. 2. Monte Carlo time series for simulated tempering run.

\section{Results and discussion}

The folded state of tryptophan zipper-I (trpzip-I) molecule with 12-residue, SWTWEGNKWTWK, is a cooperatively formed stable $\beta$-hairpin motif with hydrophobic core of packed side chains resolved by NMR experiments in aqueous solution [11]. We have given the 3D structures of trpzip molecule obtained from PDB NMR structure (1le0.pdb) and our global minimum of simulated tempering simulations in Fig. 3(a) and (b) respectively. Global energy minimum (GEM) state consists of two $\beta$-strands connected by a turn with six hydrogen bonds. Rootmean-square deviations from the reference PDB file are $1.30 \AA$ and $3.35 \AA$ for backbone and heavy atom, respectively. RMSD for $C_{\alpha}$ atoms is $1.43 \AA$ and very close to backbone rmsd value as expected. Heavy atom rmsd which is calculated from non-hydrogen atoms may be important due to topologically distinct states in $\beta$-strands. The $\approx 2 \AA$ difference between backbone and heavy atom (non-hydrogen) rmsd values is not surprising for this molecule and mainly related to the conformational freedom of side chain atoms in the solvent environment. Moreover, no constraint was applied on dihedral angles and this short peptide is composed of relatively large amino acid side chains like tryptophan, glutamic acid, and lysine. Consequently, this $\approx 2 \AA$ difference is also conserved at other temperatures as seen in Table 2. In Fig. 4, we display as a showcase the Ramachandran plot constructed for alanine-dipeptide a single-residue peptide having largest possible conformational space of an amino acid restricted by steric clashes. Dark and gray regions show the allowed $\phi$ and $\psi$ backbone dihedral angles and small squares show the dihedral angles of our reference NMR structure obtained from data file 1le0.pdb. We have also given the backbone dihedral angles of the reference structure and our GEM conformation in Table 1 in order to see the structural similarity of these two conformations clearly. 1st, 6th and 7th residues of both structures are in the non-allowed region of the Ramachandran map. It is interesting that the dihedral angles of most residues are in good agreement, but there are drastic differences in dihedral angles of the residues which fall out of the allowed region. Amino terminal of the first residue was not capped with any group, hence the 1st residue is the most flexible one in the chain with high degrees of freedom and has low-level geometrical constraints as compared to other residues in solvent conditions. But the residues which are close to the chain center are expected to form more compact structures than the other residues due to steric clashes. In this special motif, 6th and 7th residues occupy the link between two $\beta$-strands to form the so-called type II' Gly-Asn $\beta$-turn and their backbone dihedral angles are enforced to have non-allowed values by the other forces, primarily the strong hydrogen bonds in the chain and hydrophobic forces applied by the solvent. Most side chains in the molecule are of strongly hydrophobic character and lead to form a hydrophobic core. In solvent conditions, water bridges, which are the hydrogen bonds between chain and water molecules, are effective on the stabilization of the chain. But our implicit solvent environment prevents the observation of water bridge formation processes.

A visualization of the whole free energy landscape as functions of temperature and the conformational quantities provides a detailed picture of the folding pathway. In Fig. 5, free energy and probability profiles vs temperature and $\beta$-strand content are shown. Free energy was calculated by counting the histogram values (number of sampled conformations) for $\beta$-strand content at definite temperatures using the formula:

$$
F(\beta, T)=-R T \ln p(\beta, T),
$$


Table 1

Backbone dihedral angles of the reference conformation (1le0.pdb) and our GEM (global energy minimum) structure obtained from simulated tempering run

\begin{tabular}{|c|c|c|c|c|}
\hline & \multicolumn{2}{|c|}{ 11e0.pdb } & \multicolumn{2}{|l|}{ GEM } \\
\hline & $\bar{\phi}$ & $\psi$ & $\bar{\phi}$ & $\psi$ \\
\hline$S^{*}$ & 0 & 151 & 0 & 103 \\
\hline W & -70 & 116 & -87 & 120 \\
\hline $\mathrm{T}$ & -117 & 154 & -117 & 134 \\
\hline W & -94 & 129 & -93 & 125 \\
\hline $\mathrm{E}$ & -113 & 46 & -120 & 59 \\
\hline$G^{*}$ & 89 & -56 & 52 & 110 \\
\hline $\mathrm{N}^{*}$ & -132 & -12 & -124 & 37 \\
\hline K & -133 & 159 & -152 & 152 \\
\hline W & -83 & 158 & -91 & 141 \\
\hline $\mathrm{T}$ & -151 & 146 & -133 & 129 \\
\hline W & -91 & 131 & -99 & 125 \\
\hline K & -101 & 113 & -118 & 112 \\
\hline
\end{tabular}

Residues in the non-allowed region are indicated by stars.

Table 2

Average values of several structural and geometric quantities of tryptophan zipper-I molecule at each temperature range

\begin{tabular}{|c|c|c|c|c|c|}
\hline$T(\mathrm{~K})$ & $\langle\beta$-strand $\rangle$ & $\langle\alpha$-helix $\rangle$ & $\left\langle R_{g y}\right\rangle(\AA)$ & $\left\langle r m s d_{b b}\right\rangle(\AA)$ & $\left\langle r m s d_{h v}\right\rangle(\AA)$ \\
\hline 201 & 0.48 & 0.02 & 6.6 & 1.47 & 3.54 \\
\hline 216 & 0.45 & 0.03 & 6.6 & 1.68 & 3.76 \\
\hline 231 & 0.41 & 0.04 & 6.7 & 2.03 & 4.12 \\
\hline 249 & 0.36 & 0.05 & 6.8 & 2.60 & 4.67 \\
\hline 267 & 0.30 & 0.08 & 6.9 & 3.46 & 5.47 \\
\hline 287 & 0.23 & 0.09 & 7.3 & 4.50 & 6.42 \\
\hline 308 & 0.19 & 0.09 & 7.8 & 5.48 & 7.30 \\
\hline
\end{tabular}

with the probability distribution of the $\beta$-strand content

$$
p(\beta, T)=\int \mathcal{D} \xi \delta(\beta-\beta(\xi)) \mathrm{e}^{-\beta E(\xi)},
$$

where the integral runs over all possible conformations $\xi$. There exist two minima on the free energy surface, this situation is also supported by the probability profile with two high probability regions showing a two-state picture. Global minimum of free energy landscape lies about $200-250 \mathrm{~K}$ with $0.3-0.6 \mathrm{high} \beta$-strand content and $5.8 \mathrm{kcal} / \mathrm{mol}$ energy value. The other local minimum is at about $300-340 \mathrm{~K}$ with $0.1-0.2$ relatively low $\beta$-strand structure and $7.5 \mathrm{kcal} / \mathrm{mol}$ energy. Global minimum energy valley corresponds to a native $\beta$-hairpin structure with approximately $\approx 1 \AA$ backbone and $\approx 3 \AA$ heavy atom root-mean-square deviations from the reference PDB structure as seen in Fig. 6 . The local minimum seen in Fig. 5 is a non-native (unfolded) state of $\beta$-hairpin with 6-7 $\AA$ and 8-9 $\AA$ backbone and heavy atom root-mean-square deviations, respectively.

In Table 2, we have shown the average values of all structural quantities for each temperature range. $\alpha$-helix content of the chain changes from 0.02 to 0.09 from low temperatures to higher ones, while $\beta$-strand content changes from 0.48 to 0.19 on average. That means the unfolded state of the system can be regarded as a random-coil containing $9 \%$ $\alpha$-helix and $19 \% \beta$-strand structure, although the increase in the $\alpha$-helix content with temperature is remarkable. The radius of gyration with unit mass changes from 6.6 to $7.8 \AA$ while a remarkable change is noticed in RMSD with $\approx 4 \AA$ at the same temperature scale. Radius of gyration is a global geometrical quantity, so that there is no drastic change in compactness of the molecule between folded and unfolded states. This situation is not very surprising for such a small peptide and may also be attributed to the fact that the folding-unfolding transition in $\beta$-hairpins is not very sharp like the helix-coil transitions in peptides and nucleic acids. There is also a noteworthy point that, the backbone rmsd value changes from 1.47 to $5.48 \AA$ from low temperatures to higher ones, while heavy atom rmsd value goes from 3.54 to $7.30 \AA$. Conformational transitions are characterized by the changes of the backbone dihedral angles 

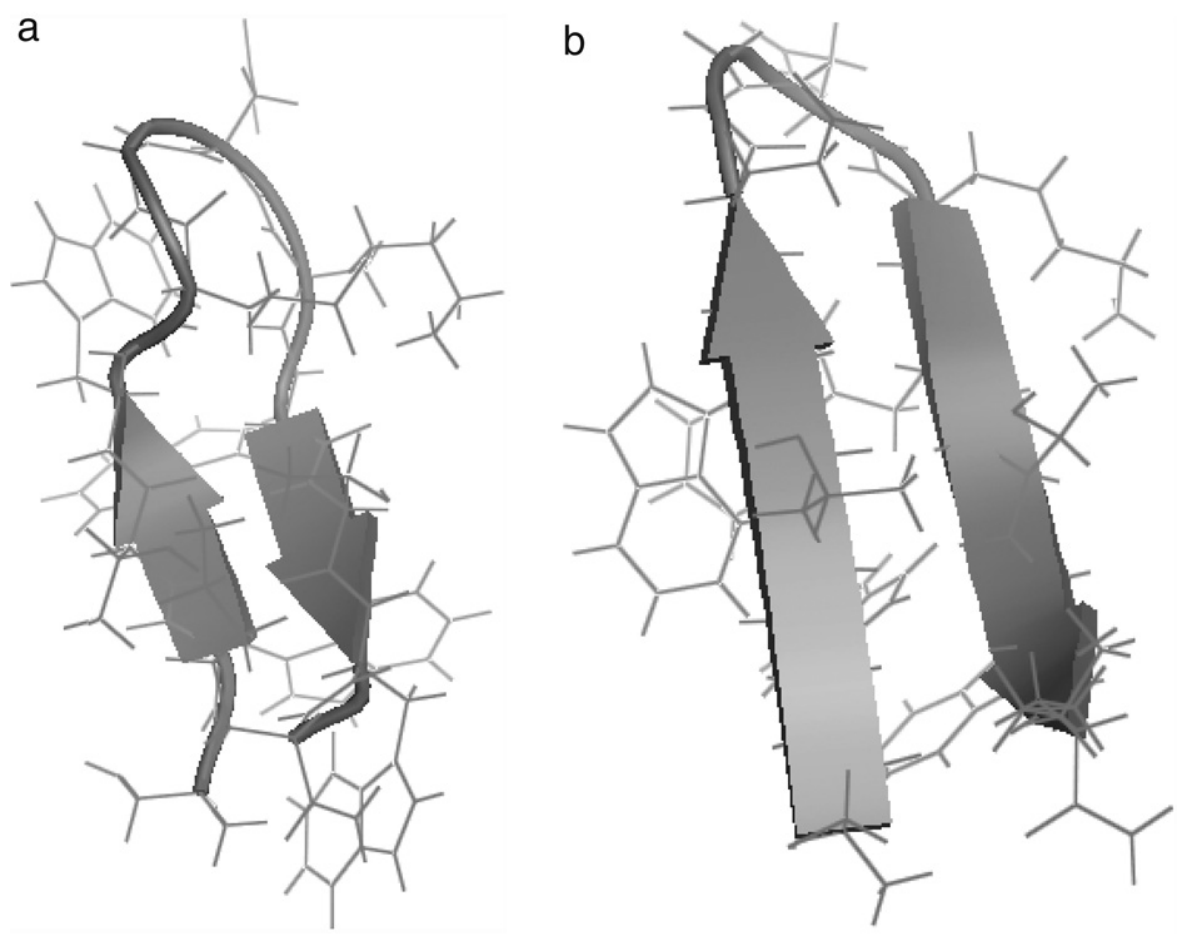

Fig. 3. (a): 3D structure of tryptophan zipper molecule obtained from pdb file (1le0.pdb) and (b): our global minimum energy configuration obtained from simulated tempering run with a $1.30 \AA$ backbone rmsd deviation from the reference structure shown in (a).

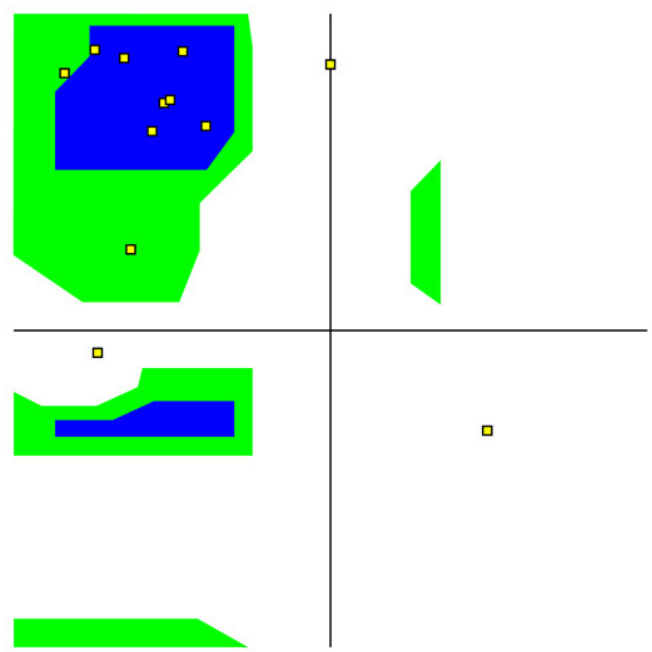

Fig. 4. Ramachandran plot of the reference pdb structure obtained from data file 1le0.pdb. Horizontal axis is $\phi$, vertical axis is $\psi$ dihedral angles both in the range $(-180,180)$.

and geometrical constraints strongly apply on backbone atoms. Side chains have higher molecular degrees of freedom than the backbone atoms in both folded and unfolded structures. Consequently, the backbone atoms show a larger deviation from the reference structure in the studied temperature range.

Unfolded states of proteins have attracted less attention than the folded state, because the biologically active state corresponds to the native structure [31]. Zagrovic et al. studied the unfolded ensemble of tryptophan zipper molecule and found $6.6 \AA$ radius of gyration for native structure and $\approx 3.5 \AA$ average RMSD from the native experimental structure [32]. They have also found native-like geometries for the unfolded state by averaging out the unfolded 

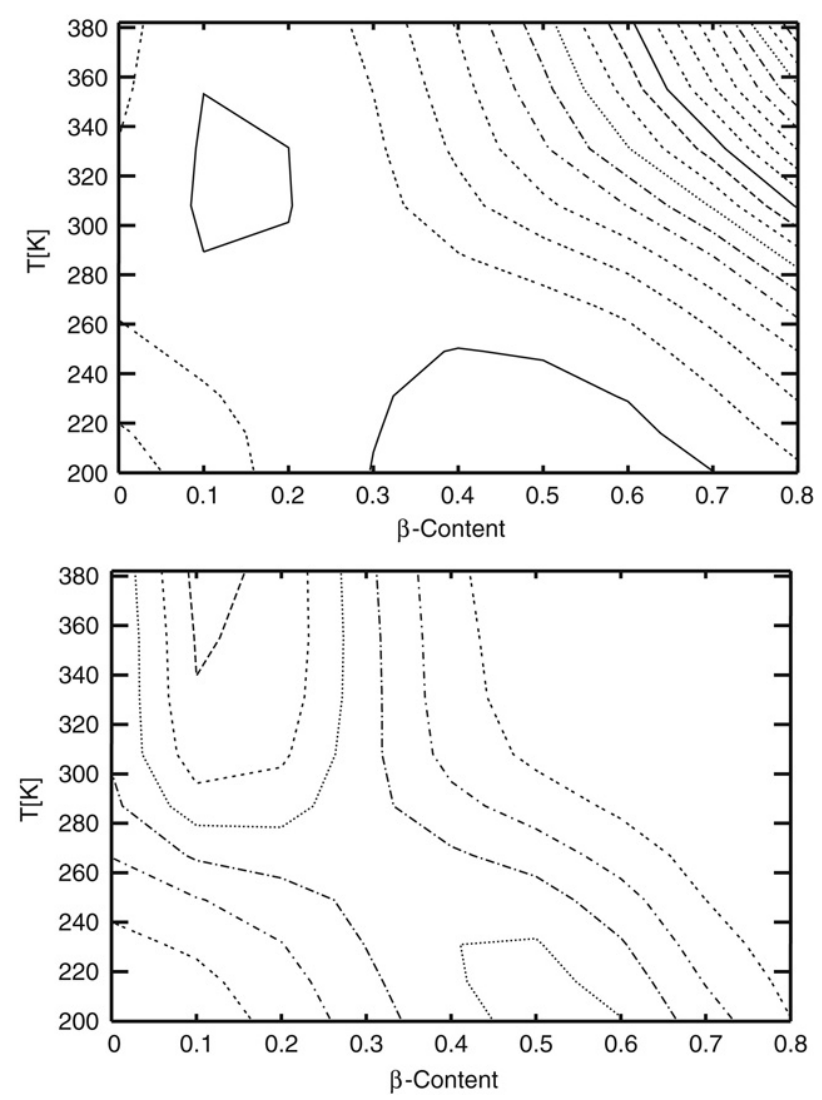

Fig. 5. Free energy (upper) and probability (lower) profiles vs temperature and $\beta$-strand content as a contour graph. Contours are spaced at $4 \mathrm{kcal} / \mathrm{mol}$ and $5 \times 10^{-2}$ intervals, respectively.

Table 3

$\%$ populations of the folded conformations at each temperature studied in this work

\begin{tabular}{ll}
\hline$T(\mathrm{~K})$ & $\%$ populations \\
\hline 201 & 89 \\
216 & 85 \\
231 & 81 \\
249 & 71 \\
267 & 54 \\
287 & 44 \\
308 & 29 \\
331 & 25 \\
355 & 27 \\
\hline
\end{tabular}

ensemble in view of "mean structure hypothesis" [33]. Contradictory data were reported in the literature about the formation of $\alpha$-helical structures in the unfolded state. Bolhuis did not find any $\alpha$-helical structure in unfolded $\beta$ hairpin when using explicit solvent [34]. Irbäck et al. have found two local minima in free energy surface, one of which corresponds to the non-native $\beta$-hairpin with $\approx 5 \AA$ RMSD while the other one corresponds to the $\alpha$-helix [16]. These differences are mainly due to the potential energy functions used, rather than the simulation technique.

In Table 3, we have given the $\%$ populations of folded $\beta$-hairpin conformations which have 0.3 or more $\beta$-strand content. At ground state $89 \%$ of the whole population is native $\beta$-hairpin while only $27 \%$ is native structure at high temperature regime. In a previous work, $78 \%$ folded secondary structure was found in native equilibrium population 

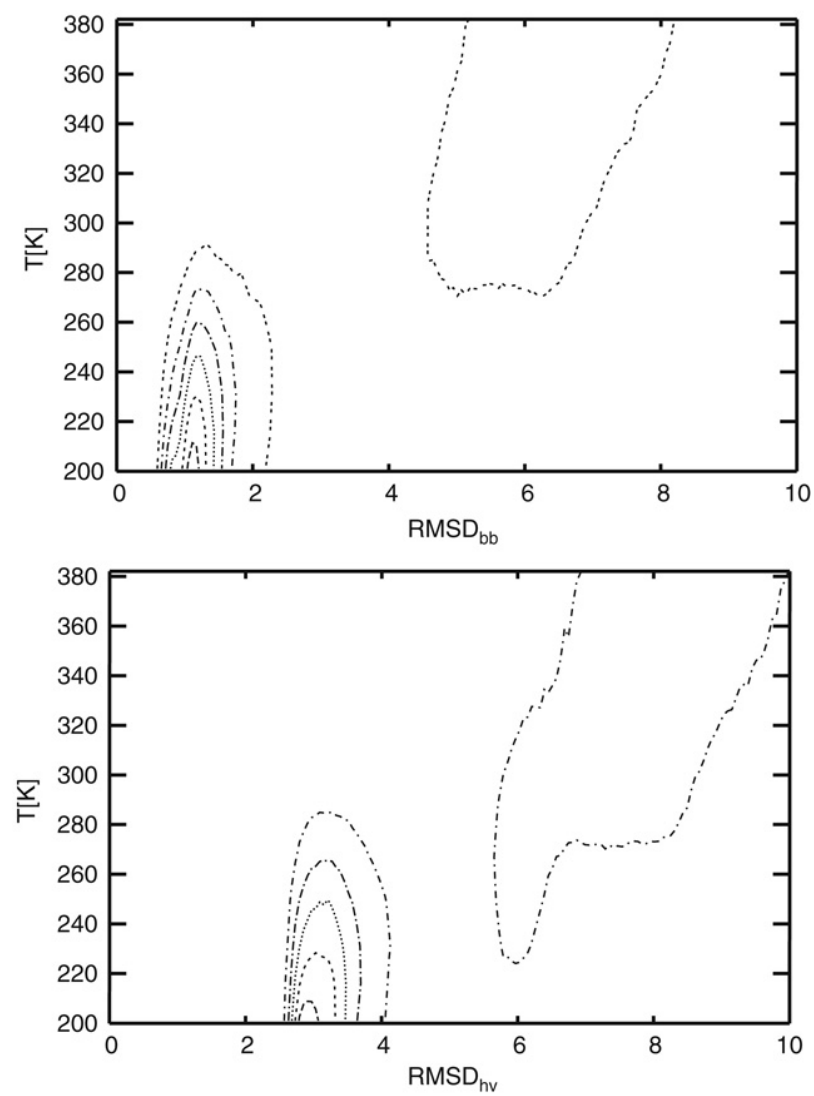

Fig. 6. Probability profiles vs temperature and backbone (upper) and heavy atom (lower) RMSD (in $\AA$ ) as a contour graph. Contours are spaced at $1 \times 10^{-2}$ intervals.

of trpzip molecule by molecular dynamics (MD) simulations [32]. In another study, 27\% native-like structure was found in unfolded ensemble [33].

At about $280 \mathrm{~K}$ temperature, pseudophases of folded and unfolded states coexist together as seen in Fig. 6 indicating a transition between distinct macrostates. Hence melting temperature for $\beta$-hairpin motif must be between the 270 and $290 \mathrm{~K}$ region where the native and non-native states have equal probability. Thermal unfolding temperature of trpzip-I molecule was determined as $323 \mathrm{~K}$ experimentally [11]. The relatively low transition temperature that was obtained may be attributed to well-known force field uncertainties [35].

Another study using the same force field investigated the artificial peptide beta3s with 20 amino acids on the basis of parallel tempering simulations [36]. The native structure of this molecule was a three-stranded $\beta$-sheet in aqueous solution. They found only $10 \%$ native-like population in $274 \mathrm{~K}$ ensemble and most conformations have a relatively large backbone rmsd value $\approx 6 \AA$ from the reference NMR structure. Their results in the 274-374 K temperature range indicate a collapse into dense structures rather than a conformational transition. This situation shows a lower efficiency in comparison to our work. This can be due to the simulation technique used and the studied peptide chain with larger size. It should also be noted that the free energy surfaces of $\beta$-sheet structures show more complex profiles than the $\alpha$-helical peptides.

\section{Conclusion}

In this study, we have investigated the folding behavior of tryptophan zipper-I molecule which folds into a stable $\beta$-hairpin motif in aqueous solution. We have used the simulated tempering algorithm in order to get equilibrium occupation statistics of the molecule at various temperatures. Our global minimum energy conformation obtained from simulated tempering run has a $1.4 \AA$ backbone rmsd value from the reference pdb structure. Two minima were found on free energy landscape, one of which corresponds to native $\beta$-hairpin with $1 \AA$ rmsd while the other one is 
a non-native structure with $\approx 6 \AA$ rmsd value. Fractional folded populations of folded and unfolded states agree well with the other studies. Our results demonstrate the success of the used force field and the simulation algorithm in the investigation of the structure and thermodynamics of folding proteins with rough energy landscape.

\section{Acknowledgments}

This project was partially supported by Hacettepe University Scientific Research Fund through project numbers 06.T09.604.008 and 02.02.602.010. T.Ç. acknowledges the Turkish Academy of Sciences (TÜBA).

\section{References}

[1] U.H.E. Hansmann, Y. Okamoto, J. Phys. Chem. B 102 (1998) 653.

[2] Y. Peng, U.H.E. Hansmann, Biophys. J. 82 (2002) 3269.

[3] G. Gökoğlu, T. Çelik, Internat. J. Modern Phys. C 17 (2006) 235.

[4] M.J. Sippl, G. Nemethy, H.A. Scheraga, J. Phys. Chem. 88 (1984) 6231.

[5] B.R. Brooks, R.E. Bruccoleri, B.D. Olafson, D.J. States, S. Swaminathan, M. Karplus, J. Comput. Chem. 4 (1983) 187.

[6] D.A. Case, T.E. Cheatham, T. Darden, H. Gohlko, R. Luo, K.M. Merz, A. Onufriev, C. Simmerling, B. Wang, R. Woods, J. Comput. Chem. 26 (2005) 1668.

[7] G. Gökoğlu, M. Bachmann, T. Çelik, W. Janke, Phys. Rev. E 74 (2006) 041802.

[8] H. Arkın, T. Çelik, Eur. Phys. J. B 30 (2002) 577.

[9] M. Bachmann, W. Janke, Phys. Rev. Lett. 91 (2003) 208105.

[10] M. Bachmann, W. Janke, J. Chem. Phys. 120 (2004) 6779.

[11] A.G. Cochran, N.J. Skelton, M.A. Starovasnik, Proc. Natl. Acad. Sci. USA 98 (2001) 5578.

[12] A. Irbäck, F. Potthast, J. Chem. Phys. 103 (1995) 10298.

[13] U.H.E. Hansmann, Y. Okamoto, Phys. Rev. E 54 (1996) 5863.

[14] A. Irbäck, S. Mohanty, J. Comput. Chem. 27 (2006) 1548.

[15] A. Irbäck, S. Mohanty, Biophys. J. 88 (2005) 1560.

[16] A. Irbäck, B. Samuelsson, F. Sjunnesson, S. Wallin, Biophys. J. 85 (2003) 1466.

[17] E. Eisenmenger, U.H.E. Hansmann, S. Hayryan, C.K. Hu, Comput. Phys. Commun. 138 (2001) 192.

[18] E. Lindahl, B. Hess, D. van der Spoel, J. Mol. Model. 7 (2001) 306.

[19] T. Schaumann, W. Braun, K. Wüthrich, Biopolymers 29 (1990) 679.

[20] W. Nadler, U.H.E. Hansmann, Phys. Rev. E 75 (2007) 026109.

[21] S. Trebst, M. Troyer, U.H.E. Hansmann, J. Chem. Phys. 124 (2006) 174903.

[22] U.H.E. Hansmann, Y. Okamoto, J. Comput. Chem. 18 (1997) 920.

[23] B.A. Berg, U.H.E. Hansmann, Y. Okamoto, J. Phys. Chem. 99 (1995) 2236.

[24] B.A. Berg, T. Neuhaus, Phys. Lett. B 267 (1991) 249.

[25] S. Kirkpatrick, C.D. Gelatt Jr., M.P. Vecchi, Science 220 (1983) 671.

[26] K. Hukushima, K. Nemoto, J. Phys. Soc. Japan 65 (1996) 1604.

[27] G. Gökoğlu, T. Çelik, Internat. J. Modern Phys. C 18 (2007) 91.

[28] U.H.E. Hansmann, Chem. Phys. Lett. 281 (1997) 140.

[29] E. Marinari, G. Parisi, Eur. Phys. Lett. 19 (1992) 451.

[30] A.P. Lyubartsev, A.A. Martinovski, S.V. Shevkunov, P.N. Vorontsov-Velyaminov, J. Chem. Phys. 96 (1992) 1776.

[31] K.W. Plaxco, M. Gross, Nature Struct. Biol. 8 (2001) 659.

[32] B. Zagrovic, C.D. Snow, S. Khaliq, M.R. Shirts, V.S. Pande, J. Mol. Biol. 323 (2002) 153.

[33] B. Zagrovic, V.S. Pande, Nature Struct. Biol. 10 (2003) 955.

[34] P.G. Bolhuis, Biophys. J. 88 (2005) 50.

[35] C.-Y. Lin, C.-K. Hu, U.H.E. Hansmann, Proteins: Struct. Funct. Genet. 52 (2003) 436.

[36] S. Mohanty, U.H.E. Hansmann, Biophys. J. 91 (2006) 3573. 